\title{
Earlier Development of the Accumbens Relative to Orbitofrontal Cortex Might Underlie Risk-Taking Behavior in Adolescents
}

\author{
Adriana Galvan, ${ }^{1}$ Todd A. Hare, ${ }^{1}$ Cindy E. Parra, ${ }^{1}$ Jackie Penn, ${ }^{1}$ Henning Voss, ${ }^{1}$ Gary Glover, ${ }^{2}$ and B. J. Casey ${ }^{1}$ \\ ${ }^{1}$ The Sackler Institute for Developmental Psychobiology, Weill Medical College of Cornell University, New York, New York 10021, and ${ }^{2}$ Department of \\ Radiology and Neurosciences Program, Center for Advanced Magnetic Resonance Technology at Stanford University, Stanford, California 94305
}

\begin{abstract}
Adolescence has been characterized by risk-taking behaviors that can lead to fatal outcomes. This study examined the neurobiological development of neural systems implicated in reward-seeking behaviors. Thirty-seven participants (7-29years of age) were scanned using event-related functional magnetic resonance imaging and a paradigm that parametrically manipulated reward values. The results show exaggerated accumbens activity, relative to prefrontal activity in adolescents, compared with children and adults, which appeared to be driven by different time courses of development for these regions. Accumbens activity in adolescents looked like that of adults in both extent of activity and sensitivity to reward values, although the magnitude of activity was exaggerated. In contrast, the extent of orbital frontal cortex activity in adolescents looked more like that of children than adults, with less focal patterns of activity. These findings suggest that maturing subcortical systems become disproportionately activated relative to later maturing top-down control systems, biasing the adolescent's action toward immediate over long-term gains.
\end{abstract}

Key words: accumbens; adolescent; reward; development; orbital frontal cortex; risk-taking

\section{Introduction}

Onset of substance abuse often occurs during the increased risktaking period of adolescence (Silveri et al., 2004). Little is known to date about neurobiological factors that may predispose adolescents to increased risk-taking behaviors. In adults, mesolimbic regions have been implicated in reward (Knutson et al., 2001; Elliott et al., 2003; McClure et al., 2004), risk-taking (Kuhnen and Knutson, 2005), and addiction (Hyman and Malenka, 2001; Volkow et al., 2004), but less is known about the development of these systems. The purpose of this study was to test the hypothesis that adolescence is a developmental period of increased responsivity to reward relative to childhood and adulthood. Specifically, we examined whether differences in the development of subcortical [e.g., nucleus accumbens (NAcc)] relative to prefrontal [e.g., orbital frontal cortex (OFC)] regions may characterize this period of development to help explain the increase in risk-taking behavior.

Adolescence is characterized by continued structural and functional development of frontostriatal circuitry implicated in behavioral regulation. Periadolescent rats show increases in reward-related dopamine transmission in the striatum (Laviola

Received Jan. 5, 2006; revised May 15, 2006; accepted May 25, 2006.

This work was supported in part by National Institute on Drug Abuse Grants R01 DA18879 and R21 DA15882, National Institute of Mental Health Grant P50 MH62196 (B.J.C.), and National Eye Institute Grant T32 EY07138 fellowship (A.G.). We gratefully acknowledge all of the participants and their families for participating in this study and three anonymous reviewers.

Correspondence should be addressed to either Adriana Galvan or B. J. Casey, 1300 York Avenue, Box 140, New York, NY 10021. E-mail: adg2006@med.cornell.edu or bjc2002@med.cornell.edu. D0I:10.1523/JNEUROSCI.1062-06.2006

Copyright $\odot 2006$ Society for Neuroscience $\quad$ 0270-6474/06/266885-08\$15.00/0 et al., 1999), and nonhuman primates show increased dopaminergic innervation in the prefrontal cortex (PFC) (Rosenberg and Lewis, 1994, 1995). Human imaging studies show frontostriatal region changes (Giedd et al., 1999; Sowell et al., 1999; Casey et al., 2005) that seem to parallel increased cognitive control (Casey et al., 1997; Rubia et al., 2000; Luna et al., 2001; Luna and Sweeney, 2004; Steinberg, 2004). These changes appear to show activation shifts of prefrontal regions from diffuse to more focal recruitment over time (Casey et al., 1997; Bunge et al., 2002; Moses et al., 2002; Durston et al., 2006). Neuroimaging studies cannot definitively characterize the mechanism of such developmental changes (e.g., synaptic pruning, myelination). However, these volume and structural changes may reflect refinement and finetuning of reciprocal projections from these brain regions during maturation. Thus, this interpretation is only speculative.

Recently, neuroimaging studies have begun to examine reward-related processing in adolescents and have shown NAcc activation as shown in adults (Bjork et al., 2004; May et al., 2004; Ernst et al., 2005). However, the results have been mixed as to how adolescents and adults differ in activity. These studies have focused primarily on the region of the accumbens rather than OFC in examining changes. Furthermore, little attention has been given to characterizing NAcc and OFC development from childhood through adulthood. Tracking this development provides additional constraints on whether changes reported in adolescence are specific to this period of development or reflect maturational linear changes.

Here, we used functional magnetic resonance imaging (fMRI) to examine behavioral and neural responses to reward value ma- 
nipulations across development. We focused on the NAcc and OFC given previous reports in animal (Hikosaka and Watanabe, 2000; Pecina et al., 2003), imaging (O'Doherty et al., 2001; Zald et al., 2004), and addiction (Hyman and Malenka, 2001) studies implicating them in reward-related learning. Based on rodent models (Laviola et al., 1999; Spear, 2000) and previous imaging work (Ernst et al., 2005), we hypothesized that, relative to children and adults, adolescents would show an exaggerated accumbens response suggestive of refined focal activations within the accumbens during this period, in concert with less mature activations in top-down PFC regions.

\section{Materials and Methods}

Participants. Sixteen children (seven females; aged $7-11$, with mean age of 9.8 years), 13 adolescents (six females; aged 13-17, with mean age of 16 years), and 12 right-handed healthy adults (six females; aged 23-29, with mean age of 25 years) participated in the fMRI experiment. A separate statistical analysis on the adult data was reported previously (Galvan et al., 2005). Three children and one adolescent were excluded from the analysis due to excessive motion $(>2 \mathrm{~mm}$ ). Motion was $>0.5$ voxels $(1.56 \mathrm{~mm}$ ) in any direction for two subjects (one child and one adult) included in the analysis. Eliminating these subjects from the analysis did not change the results and the different age groups did not differ significantly in in-plane motion (adults: $x=0.48, y=0.76, z=0.49$; adolescents: $x=0.26, y=0.58, z=0.45$; children: $x=0.18, y=0.76, z=$ 0.36 ). Subjects had no history of neurological or psychiatric disorder and each gave informed consent (parental consent and child assent for adolescents and children) for a protocol approved by the Institutional Review Board of Weill Cornell Medical College of Cornell University. The experiment on adolescents and children was simulated in a mock scanner before the experiment, in which they were exposed to the sounds they would hear during the actual experiment.

Experimental task. Participants were tested using an adapted version of a delayed response two-choice task previously used in nonhuman primates (Cromwell and Schultz, 2003) and described previously (Galvan et al., 2005) in an event-related fMRI study (Fig. 1). In this task, three cues (counterbalanced) were each associated with a distinct reward value. Subjects were instructed to press either their index or middle finger to indicate the side on which a cue appeared when prompted, and to respond as quickly as possible without making mistakes.

The stimulus parameters were as follows. One of three pirate cartoon images was presented in pseudorandom order on either the left or right side of a centered fixation for $1000 \mathrm{~ms}$ (Fig. 1). After a $2000 \mathrm{~ms}$ delay, subjects were presented with a response prompt of two treasure chests on both sides of the fixation $(2000 \mathrm{~ms})$ and instructed to press a button with their right index finger if the pirate was on the left side of the fixation or their right middle finger if the pirate was on the right side of the fixation. After another $2000 \mathrm{~ms}$ delay, reward feedback of either a small, medium, or large amount of coins was presented in the center of the screen (1000 $\mathrm{ms})$. Each pirate was associated with a distinct reward amount. There was a $12 \mathrm{~s}$ intertrial interval (ITI) before the start of the next trial. Total trial length was $20 \mathrm{~s}$. Subjects were not rewarded if they failed to make a response or if they made an error; in both cases, they received an error message at the time they would normally receive reward feedback.

Subjects were guaranteed $\$ 50$ for participation in the study and were told they could earn up to $\$ 25$ more, depending on performance (as indexed by reaction time and accuracy) on the task. Although the reward amounts were distinctly different from one another, the exact value of each reward was not disclosed to the subject, because during pilot studies, subjects reported counting the money after each trial and we wanted to avoid this possible distraction. Stimuli were presented with the integrated functional imaging system (PST, Pittsburgh, PA) using a liquid crystal display video display in the bore of the magnetic resonance (MR) scanner and a fiber optic response collection device.

The experiment consisted of five runs of 18 trials (six each of small, medium, and large reward trials), which lasted 6 min and $8 \mathrm{~s}$ each. Each run had six trials of each reward value presented in random order. At the end of each run, subjects were updated on how much money they had earned during that run. The amount of money earned was consistent across all subjects and all received a continuous schedule of reinforcement (rewarded on $100 \%$ of trials). Before beginning the experiment, subjects were shown the actual money they could earn to ensure motivation. They received detailed instructions that included familiarization with the stimuli used. For instance, subjects were shown the three cues and three reward amounts they would be seeing during the experiment. They were not told how the cues related to the rewards. We explicitly emphasized that there were three amounts of reward, one being small, another medium, and another large. These amounts are visually obvious in the experiment because the number of coins in the stimuli increases with increasing reward. Only one subject could articulate the association between specific stimuli and reward amounts, when asked explicitly about this association during debriefing of the subject at the end of the experiment.

Image acquisition. Imaging was performed using a 3T General Electric (Milwaukee, WI) MRI scanner using a quadrature head coil. Functional scans were acquired using a spiral in and out sequence (Glover and Thomason, 2004). The parameters included the following: repetition time (TR), $2000 \mathrm{~ms}$; echo time (TE), $30 \mathrm{~ms} ; 64 \times 64$ matrix; $295 \mathrm{~mm}$ coronal slices; $3.125 \times 3.125 \mathrm{~mm}$ in-plane resolution; flip, $90^{\circ}$ for 184 repetitions, including four discarded acquisitions at the beginning of each run. Anatomical T1-weighted in-plane scans were collected (TR, 500; TE, min; $256 \times 256$; field of view, $200 \mathrm{~mm}$; $5 \mathrm{~mm}$ slice thickness) in the same locations as the functional images in addition to a threedimensional data set of high-resolution spoiled gradient-recalled acquisition in a steady state images (TR, 25; TE, 5; $1.5 \mathrm{~mm}$ slice thickness; 124 slices).

Image analysis. The Brainvoyager QX (Brain Innovations, Maastricht, The Netherlands) software package was used to perform a randomeffects analysis of the imaging data. Before analysis, the following preprocessing procedures were performed on the raw images: threedimensional motion correction to detect and correct for small head movements by spatial alignment of all volumes to the first volume by 

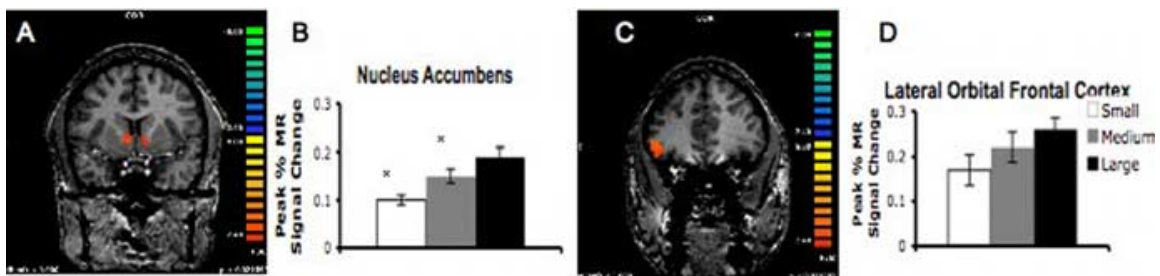

Figure 2. Localization of nucleus accumbens $(\boldsymbol{A})$ and orbital frontal cortex $(\boldsymbol{C})$ activation to reward. There was a main effect of reward value in the nucleus accumbens $(\boldsymbol{B})$ [right $(x=6, y=5, z=-2)$ and left $(x=-8, y=6, z=-2)$ ] but not in the right lateral orbital frontal cortex $(x=46, y=31, z=1)(D)$. Error bars indicate SEM. Asterisks denote significant differences between small and medium, medium and large, and small and large.

rigid body transformation, slice scan time correction (using sinc interpolation), linear trend removal, high-pass temporal filtering to remove nonlinear drifts of three or fewer cycles per time course, and spatial data smoothing using a Gaussian kernel with a $4 \mathrm{~mm}$ full width at halfmaximum. Estimated rotation and translation movements never exceeded $2 \mathrm{~mm}$ for subjects included in this analysis. Functional data were coregistered to the anatomical volume by alignment of corresponding points and manual adjustments to obtain optimal fit by visual inspection and were then transformed into Talairach space. Functional voxels were interpolated from the acquisition voxel size of $48.83 \mathrm{~mm}^{3}$ to a resolution of $1 \mathrm{~mm}^{3}$ during Talairach transformation. The NAcc and OFC were defined by Talairach coordinates in conjunction with reference to the Duvernoy brain atlas (Talairach and Tournoux, 1988; Duvernoy, 1999).

The initial omnibus general linear model (GLM) analysis included all subjects and all runs across the entire trial (relative to pretrial baseline) to determine regions sensitive to reward (NAcc and OFC). To ensure that statistical analyses were performed on the same regions for each age group, separate GLM analyses were performed. Each group showed activation in the NAcc and OFC based on a reward versus baseline contrast. Localization of these regions was further confirmed for each group separately by Talairach coordinates in conjunction with reference to the Duvernoy brain atlas (Talairach and Tournoux, 1988; Duvernoy, 1999) as described above. Previous methodological work has shown that the stereotactic registration and time course of the hemodynamic response across the ages tested in the current study are not dissimilar (Burgund et al., 2002; Kang et al., 2003). Subsequent analysis and post hoc contrasts were performed on the regions identified with this initial omnibus GLM for all groups together and then separately for each group. Last, a conjunction analysis was performed that identified the voxels that were commonly activated across all three groups, in the NAcc and OFC (supplemental Fig. 1, available at www.jneurosci.org as supplemental material). The regions of interest identified in the conjunction analysis overlapped with those identified with the initial omnibus GLM, and post hoc tests confirmed similar effects as those obtained with the above analyses.

In the whole-group analysis, the omnibus GLM was comprised of all runs across the entire trial ( 5 runs $\times 37$ subjects $=185 z$-normalized functional time courses) and was conducted with reward magnitude as the primary predictor. The predictors were obtained by convolution of an ideal boxcar response (assuming a value of 1 for the volume of task presentation and a volume of 0 for the remaining time points) with a linear model of the hemodynamic response (Boynton et al., 1996) and used to build the design matrix of each time course in the experiment. Only correct trials were included and separate predictors were created for error trials. The total number of correct trials for each group was as follows: 1130 for children $(n=13), 1061$ for adolescents $(n=12)$, and 1067 for adults $(n=12)$. The fewer number of trials for children was corrected by including an additional child subject.

Post hoc contrast analyses were then performed based on $t$ tests on the $\beta$ weights of predictors to identify a region of interest in the NAcc and OFC. Contrasts were conducted with a random-effects analysis. Time series and percent changes in MR signal, across each data point of the entire trial (18 s) relative to $2 \mathrm{~s}$ of fixation preceding trial onset (total trial duration was $20 \mathrm{~s}$ ), were calculated using event-related averaging over significantly active voxels obtained from the contrast analyses. The cal- culation of number of voxels recruited in each region by age group was based on the GLM analyses conducted on each group described above.

Corrections for multiple comparisons were based on Monte Carlo simulations, which were run using the AlphaSim program within AFNI (Cox, 1996), to determine appropriate contiguity thresholds to achieve a corrected $\alpha$ level of $p<0.01$ (Forman et al., 1995) based on a search volume of $450 \mathrm{~mm}^{3}$ for the NAcc. A corrected $\alpha$ level of $p<0.05$ in the OFC was based on a search volume of $\sim 25,400 \mathrm{~mm}^{3}$ (Forman et al., 1995). OFC activation did not survive the more stringent threshold of $p<0.01$ across groups. ${ }^{a}$

\section{Results}

\section{Imaging results}

The omnibus GLM analysis of the imaging data identified the NAcc [right $(x=6, y=5, z=-2)$ and left $(x=-8, y=6, z=$ $-2)$ ] and right OFC $(x=46, y=31, z=1)$ depicted in Figure 2, $A$ and $C$, with reward value as the primary predictor, across all subjects and runs of the experiment for the entire trial (18 s), relative to the $2 \mathrm{~s}$ intertrial interval preceding the start of the next trial (e.g., reward vs baseline contrast). Within these regions, there was a main effect of reward value $\left(F_{(2,72)}=8.424 ; p=\right.$ $0.001)$ (Fig. $2 B)$ in the NAcc, but not in the OFC $\left(F_{(2,72)}=1.3\right.$; $p=0.44$ ) (Fig. $2 D$ ). Post hoc $t$ tests on the main effect of reward for the NAcc confirmed significant differences between the large and small $\left(t_{(36)}=4.35 ; p<0.001\right)$, large and medium $\left(t_{(36)}=\right.$ $2.01 ; p<0.05)$, and medium and small $\left(t_{(36)}=2.09 ; p<0.04\right)$ rewards, with greater activation for greater rewards.

\section{Developmental differences in magnitude and extent of activity to reward}

Because the focus of this study was on how reward influences neural recruitment across development, we examined developmental differences in the magnitude and extent of accumbens and OFC activity for trials to the largest reward. The magnitude of activity was calculated as a percent change in MR signal averaged across the first $18 \mathrm{~s}$ of the trial relative to the intertrial interval of fixation immediately preceding the trial $(2 \mathrm{~s})$, which was averaged across the entire experiment ( 90 trials $=900$ scans). This calculation was performed for each group. The extent of activity was calculated as the volume of activity (number of voxels) across runs, by group, using the same contrast.

Magnitude of activity. In the accumbens and OFC, there were significant developmental differences in percent change in $\mathrm{MR}$ signal $\left(F_{(2,22)}=6.47, p<0.01 ; F_{(2,22)}=5.02, p=0.01\right.$, respectively) (Fig. $3 A, B$ ). In the accumbens, adolescents showed the greatest signal change. Post hoc tests confirmed significant differences between adolescents and children $\left(t_{(11)}=4.2 ; p=0.03\right.$ ) and between adolescents and adults $\left(t_{(11)}=5.5 ; p=0.01\right)$ in magnitude of accumbens activity. In the OFC, post hoc tests confirmed significant differences between children and adolescents $\left.t_{(11)}=4.9 ; p=0.01\right)$ and children and adults $\left(t_{(11)}=3.99 ; p=\right.$ $0.01)$. Thus, adolescents showed enhanced activity in the accumbens and this pattern differed from that in the OFC and from children and adults.

Extent of activity. There were significant developmental differ-

${ }^{a} \mathrm{Cluster}$ sizes of 6 and 10 for the accumbens and $\mathrm{OFC}$, respectively, were determined by these simulations. Cluster sizes of 8 and 10 in the adolescent and child data, respectively, survived more stringent thresholds ( $p<0.002$ and $p<0.001$, respectively). In the $\mathrm{OFC}$, cluster sizes of 14 and 18 in adolescents and children, respectively, survived more stringent thresholds ( $p<0.004$ and $p<0.001$, respectively). 


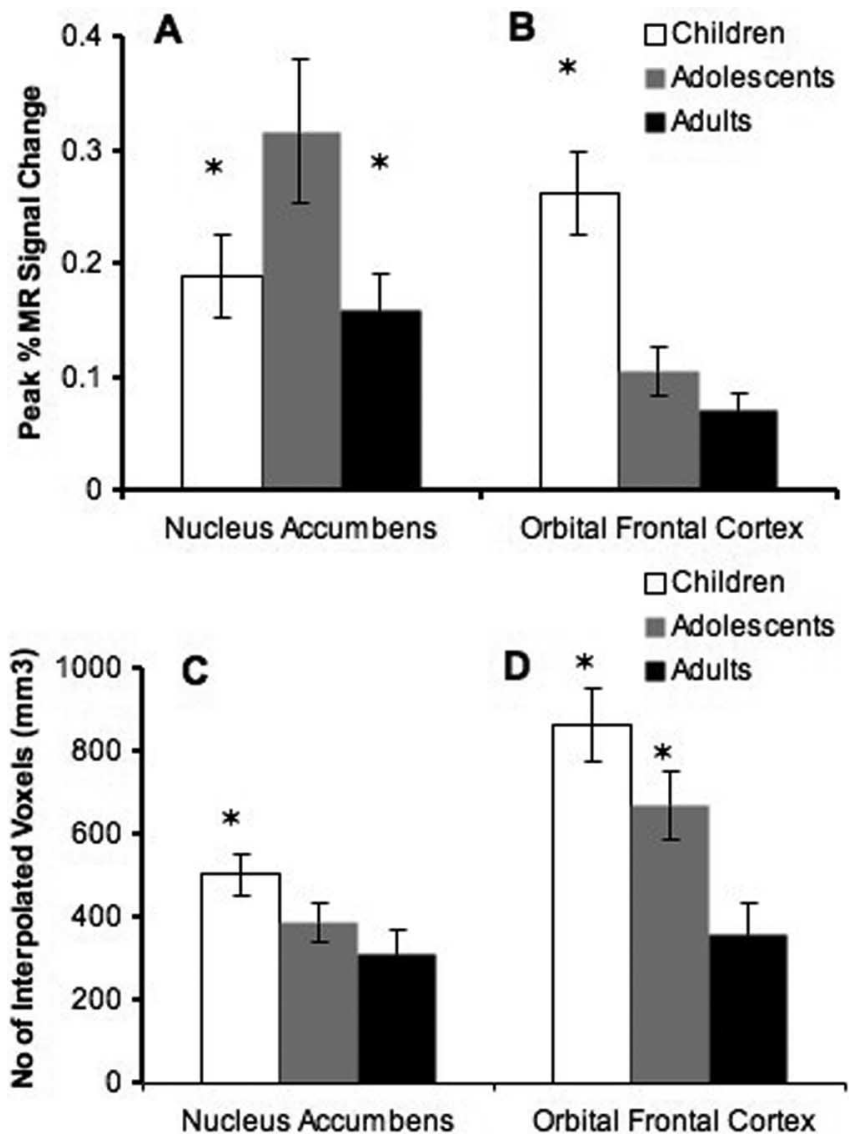

Figure 3. Magnitude and extent of accumbens and OFC activity to reward. $\boldsymbol{A}$, Adolescents showed exaggerated percent change in MR signal to large reward relative to children and adults in the accumbens. $\boldsymbol{B}$, In the $\mathrm{OFC}$, children had the greatest percent change in MR signal relative to adolescents and adults. $C$, Children showed the largest volume of activity in the accumbens relative to adolescents and adults. $\boldsymbol{D}$, Children and adolescents showed greater volume of activity in the $\mathrm{OFC}$ relative to adults. Error bars indicate SEM. Asterisks denote significant activation differences between children and adolescents and adolescents and adults in $\boldsymbol{A}$; greater activation in children relative to adolescents and adults in $\boldsymbol{B}$; greater volume of activity in children relative to adolescents and adults in $\boldsymbol{C}$; and greater volume of activity in children relative to adolescents and adolescents relative to adults in $\boldsymbol{D}$.

ences in the extent of activity in the accumbens $\left(F_{(2,22)}=4.7 ; p<\right.$ $0.02)$ and $\operatorname{OFC}\left(F_{(2,22)}=5.01 ; p=0.01\right)$. Post hoc tests confirmed the largest volume of activity in the accumbens for children $(503 \pm 43$ interpolated voxels) relative to adolescents $(389 \pm 71$ interpolated voxels) $\left(t_{(22)}=4.2 ; p<0.05\right)$ and adults $(311 \pm 84$ interpolated voxels) $\left(t_{(22)}=3.4 ; p<0.05\right)$ (Fig. $\left.3 C\right)$. Adolescents and adults did not differ $\left(t_{(22)}=0.87 ; p=0.31\right)$. For the OFC, children $(864 \pm 165$ interpolated voxels $)\left(t_{(22)}=7.1 ; p=0.01\right)$ and adolescents $(671 \pm 54)\left(t_{(22)}=5.8 ; p=0.01\right)$ showed the largest extent of activity relative to adults ( $361 \pm 45$ voxels) (Fig. $3 D)$, but there were no significant differences between children and adolescents $\left(t_{(22)}=1.8 ; p=0.07\right)$. This pattern of activity reflects protracted development of OFC relative to the NAcc (Fig. 4 , graph).

Developmental differences in temporal processing of reward value To examine differential changes in neural recruitment throughout the experiment, we examined the main effect of, and interactions with, time (early, middle, and late trials) on MR signal change in the NAcc or OFC. The effect of time was observed only in the interaction of time by group by reward in the accumbens $\left(F_{(8,136)}=3.08 ; p=0.003\right)$ and less robustly in the OFC
Normalized Extent of Activity

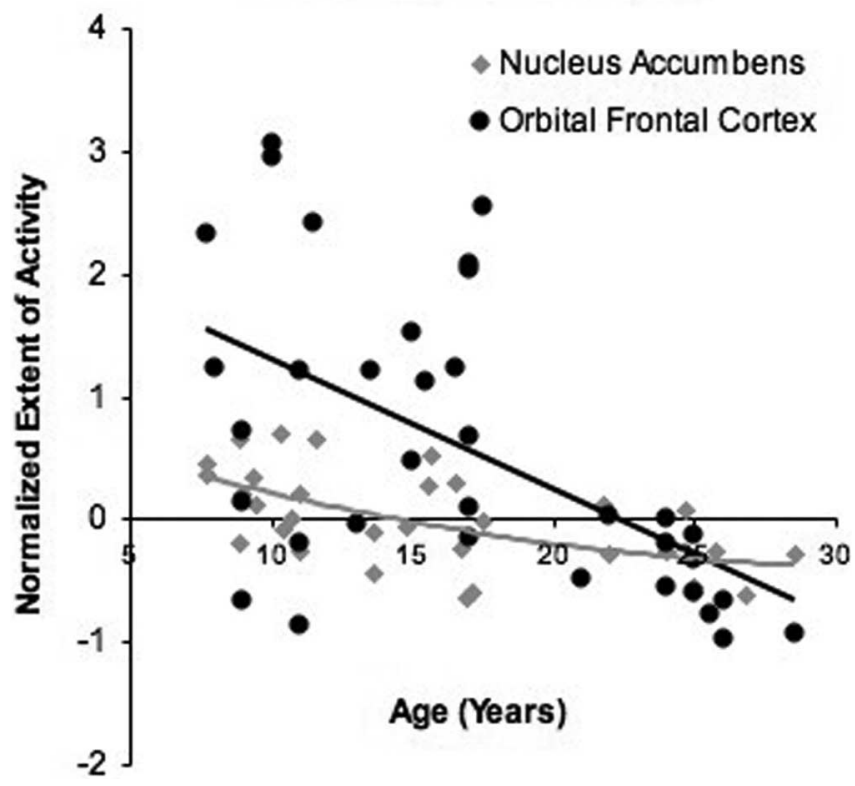

Figure 4. Normalized extent of activity measure for the nucleus accumbens and $\mathrm{OFC}$ for all subjects, adjusted for the average extent of activity $(x-$ mean/mean) for each region.

$\left(F_{(8,136)}=2.71 ; p=0.02\right)$. This interaction was driven primarily by changes occurring during the late trials of the experiment (for changes as a function of early, middle, and late trials, see supplemental Fig. 2, available at www.jneurosci.org as supplemental material). Figures 5 and 6 depict the temporal course of change in MR signal as a function of small, medium, and large reward values for late trials by group for each region. These time series show an exaggerated change in accumbens activity in adolescents relative to children or adults for the small and large reward trials that occurs $\sim 5-6 \mathrm{~s}$ after the response and the point in which all three age groups show a change in MR signal. This pattern is illustrated graphically in Figure 7 for clarity (for change in OFC activity at this time point for all three age groups, see supplemental Fig. 3, available at www.jneurosci.org as supplemental material).

\section{Behavioral results}

The effects of time on task and reward value were tested with a 5 (runs) $\times 3$ (small, medium and large reward) $\times 3$ (group) ANOVA for the dependent variables of mean reaction time for correct trials and mean accuracy. There were main effects of reward value $\left(F_{(2,72)}=9.51 ; p=0.001\right)$ and group $\left(F_{(2,220)}=4.37\right.$; $p=0.02)$ and significant interactions of reward by time $\left(F_{(8,288)}=4.176 ; p<0.001\right)$ and group by reward by time $\left(F_{(16,272)}=3.01 ; p=0.01\right)$ for mean reaction time. The main effect of reward showed that, across all subjects, mean reaction times were faster to the largest reward (mean, 515.47; SD, 178.75; $t_{(36)}=3.8 ; p<0.001$ ) relative to medium (mean, 556.89; SD, 180.53 ) or small reward (mean, 552.39; SD, 180.35). The significant interaction of reward by time was driven primarily by the three-way interaction of group by reward by time. Adults differed in mean reaction time to all three reward values by the end of the experiment (Fig. 8). Adolescents were significantly faster to the large relative to the medium and small rewards with no difference between the medium and small rewards. The children showed no significant differences in mean reaction time to the small, medium, or large rewards. There were no significant correlations 


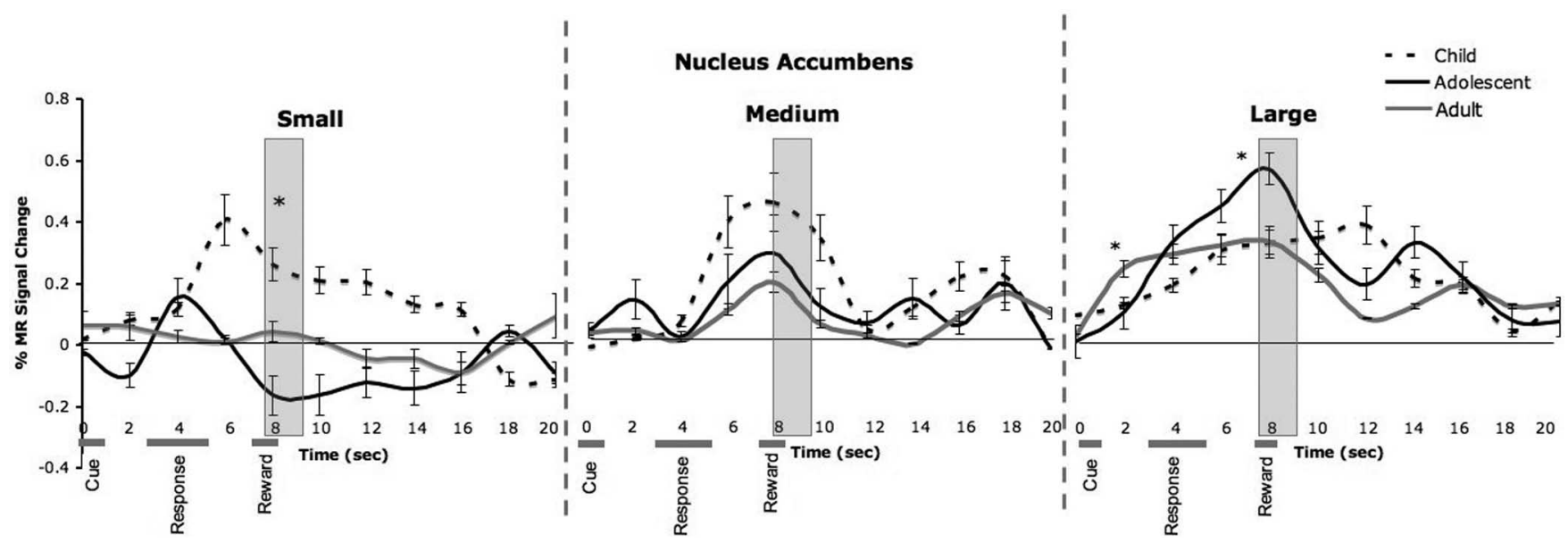

Figure 5. Temporal changes in the nucleus accumbens as a function of small, medium, and large reward values for late trials of the experiment for each age group. The gray bars correspond to the point $(s) \sim 5-6 s$ after the response. Error bars indicate SEM. Asterisks denote activation differences between groups.

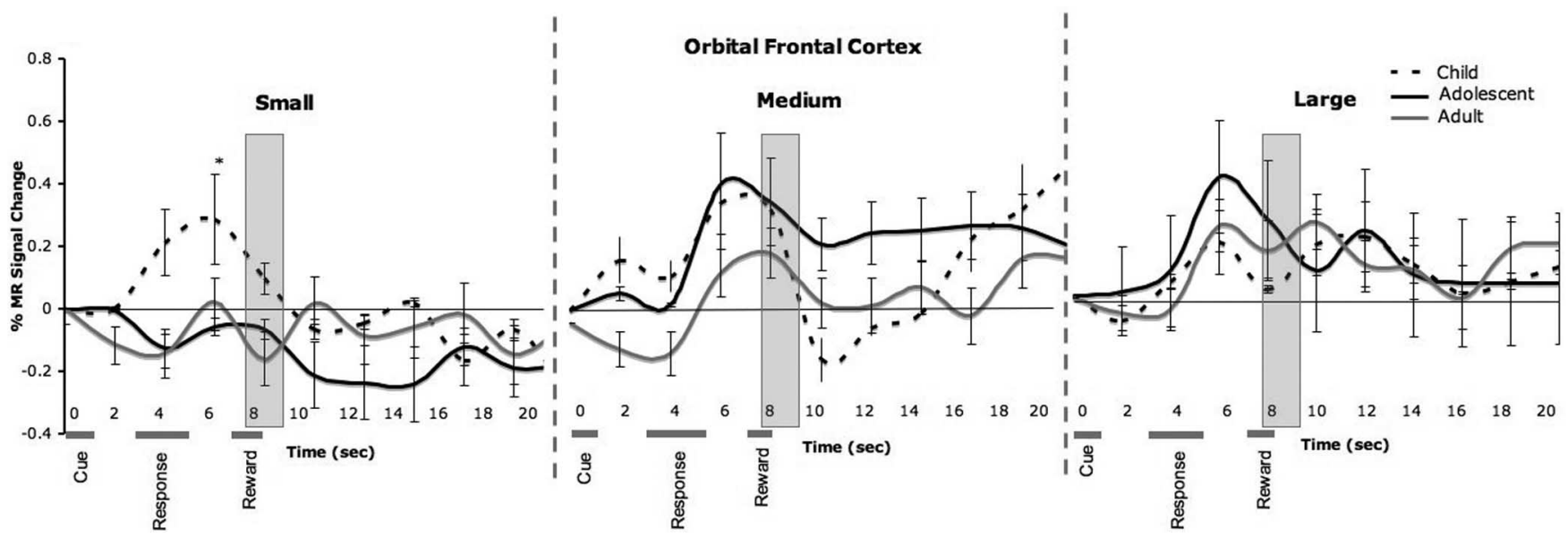

Figure 6. Temporal changes in the orbitofrontal cortex as a function of small, medium and large reward values for late trials of the experiment for each age group. The gray bars correspond to the point(s) $\sim 5-6 s$ after the response. Error bars indicate SEM. Asterisks denote activation differences between groups.

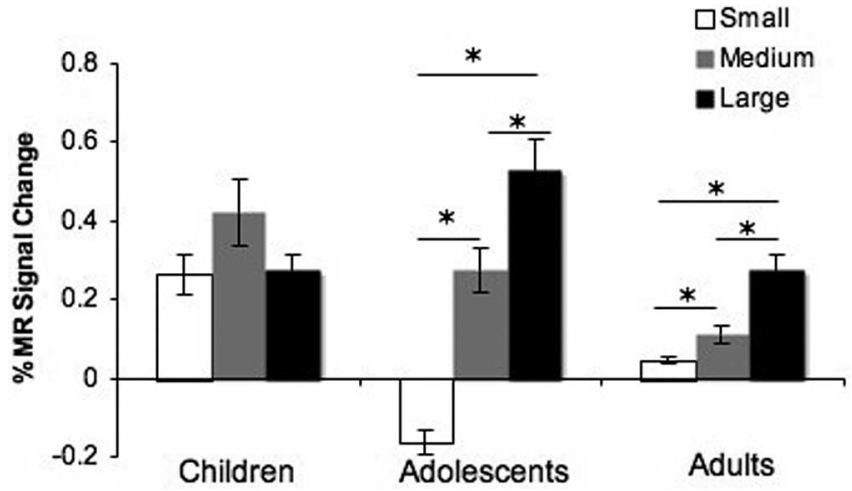

Figure 7. Percent change in MR signal $\sim 5-6 \mathrm{~s}$ after the response relative to pretrial baseline for each age group, showing an exaggerated change in accumbens activity in adolescents relative to children or adults for the small and large reward trials. Error bars indicate SEM. Asterisks denote activation differences between groups.

between mean reaction time or accuracy and accumbens or orbitofrontal activity.

There were no significant effects of reward $\left(F_{(2,72)}=0.26 ; p=\right.$ $0.40)$, group $\left(F_{(2,220)}=0.73 ; p=0.80\right)$, or time $\left(F_{(4,476)}=0.57\right.$; $p=0.44)$ or interactions for mean accuracy. All subjects had high accuracy across reward values (children: small, 96\%; medium, 98\%; large 96\%; adolescents: small, 98\%; medium, 99\%; large, 99\%; and adults: small, 98\%; medium, 99\%; large, 99\%).

\section{Discussion}

This study examined behavioral and neural responses to reward value manipulations across development. Our findings support our hypothesis that adolescents differ from children and adults in NAcc and OFC recruitment, regions previously implicated in reward processing (Knutson et al., 2001) and addiction (Volkow et al., 2004). Our results are consistent with rodent (Laviola et al., 2003) and previous developmental imaging (Ernst et al., 2005) studies of enhanced accumbens activity during adolescence. These findings suggest that different developmental trajectories for these regions may relate to the increased impulsive and risky behaviors observed during this period of development.

\section{Developmental changes in structure and function}

Enhanced accumbens activity was paralleled by a refined pattern of activity for adolescents relative to children, but similar to adults. In contrast, adolescents showed more diffuse OFC recruitment more similar to children than adults. We interpret these data to suggest that the NAcc development may precede that of the OFC during adolescence. Protracted development of 
prefrontal regions, with a transition from diffuse to focal recruitment is consistent with MRI-based neuroanatomical (Sowell et al., 1999; 2003; Gogtay et al., 2004) and fMRI studies (Casey et al., 1997, 2002; Brown et al., 2005, Durston et al., 2006) of prefrontal development (Casey et al., 2005).

Developmental changes in volume of activity within frontostriatal regions (Sowell et al., 1999) are interesting in light of known developmental processes (e.g., dendritic arborization, synaptic pruning, myelination) occurring during this period. However, neither fMRI nor MRI provide a level of analysis with which to definitively characterize the mechanism of such changes. The volume measures were used in part to constrain the interpretation of magnitude differences, but we can only speculate that our changes in volume and magnitude of activity of the NAcc and OFC reflect a fine-tuning of this circuitry with experience and development.

Differential recruitment of frontostriatal regions has been reported across several developmental fMRI studies (Casey et al., 2002; Monk et al., 2003; Thomas et al., 2004). Typically, these findings have been interpreted in terms of immature prefrontal regions rather than an imbalance between prefrontal and subcortical regions. Given evidence of prefrontal regions in guiding appropriate actions in different contexts (Miller and Cohen, 2001) immature prefrontal activity might hinder appropriate estimation of future outcomes and appraisal of risky choices, and might thus be less influential on reward valuation than the accumbens. This pattern is consistent with previous research showing elevated subcortical, relative to cortical activity when decisions are biased by immediate over long-term gains (McClure et al., 2004). Furthermore, accumbens activity has been shown to positively correlate with subsequent risk-taking behaviors (Kuhnen and Knutson, 2005).

\section{Reward-related learning across development}

One goal of this study was to characterize reward learning across development. Adults showed behavioral distinction to the three cues, with fastest responses to the large reward cue. Adolescents showed less discrete responses and children show little to no learning. Slower learning across development parallels the imaging results of protracted OFC development that may hinder associative learning between predictive events and reward outcomes. This interpretation is supported by animal (Hikosaka and Watanabe, 2000; Chudasama and Robbins, 2003; Cetin et al., 2004; Hosokawa et al., 2005) and human imaging (Elliott et al., 2000; O'Doherty et al., 2003; McClure et al., 2004; Cox et al., 2005; Galvan et al., 2005) studies showing the role of the OFC in learning and representing links between predictive events (stimuli and responses) and reward outcomes in optimizing choice behavior.

Few imaging studies of reward to date have been able to show differences in behavior as a function of reward outcome (Haruno et al., 2004; Delgado et al., 2005; Galvan et al., 2005). Here, our data suggest that reward-related neural responses influence behavioral output. Minimal behavioral variability might have precluded previous authors from determining whether different re- ward conditions bias behavioral output. One reason we were able to tease apart behavioral differences might be because our paradigm was designed to maximize behavioral responses and learning by using a continuous reinforcement schedule (Dickinson and Mackintosh, 1978; Gottlieb, 2004, 2005). Animal studies show faster learning with continuous relative to intermittent reinforcement schedules (Gottlieb, 2004) that may have explained the faster responses to large reward trials across subjects and the distinct pattern of behavior for each reward value in the adults by late trials.

\section{Rewards are relative for different contexts and ages}

Reward preference varies based on the reward context (Tversky and Kahneman, 1981; Tremblay and Schultz, 1999). Evidence from our study supports the notion that relative reward preference is exaggerated during adolescence: adolescents showed an enhanced accumbens response to the large reward and a decrease in activity to the small reward relative to other rewards and to other ages. Adolescents report greater intensity of positive feelings and more positive BOLD signal intensity than adults during a win condition (Ernst et al., 2005). The adolescents may have viewed the small reward as an omission of reward, similar to lack of an expected event at a given time, previously shown to decrease striatal activity (Davidson et al., 2004). This finding corresponded to a slowing of reaction time from early to late trials for the smaller rewards, providing additional evidence that this condition may have been perceived as more negative for adolescents. Together, these findings imply that reward perception might be influenced by changes in neural systems during adolescence (Irwin, 1993).

\section{Developmental changes may parallel changes with learning} Recently, Pasupathy and Miller (2005) showed that, in monkeys, striatal areas detected reward contingencies first, which then seemed to bias prefrontal regions into taking action. Other work has shown that the OFC seems to be implicated in linking responses with outcomes (Elliott et al., 2000; Galvan et al., 2005). However, this effect may be dependent on maturity of prefrontal systems and reciprocal connections between frontostriatal re- 
gions (Haber, 2003) that associate actions with outcome, because children and adolescents did not show learning, as indexed by mean reaction time, to the extent that adults did. It remains an open question whether the children could not learn to discriminate between the different reward values or whether they were just as happy with a small reward as a large reward.

The findings of less sensitivity in the behavioral response than in the neural response in the younger subjects may be consistent with previous learning studies showing that neural changes precede behavioral changes (Tremblay et al., 1998). The adolescents were significantly faster to larger reward trials by the end of the experiment relative to the other reward values, but the accumbens showed distinct patterns of activity to each reward value similar to adults. If this explanation were true, we might expect with additional training that the adolescents' behavioral performance would ultimately parallel the accumbens activity. Likewise, one would expect similar patterns to emerge in children, but with more extensive training.

\section{Contrasts between current and previous findings}

Although the exaggerated accumbens response in adolescents replicates those of May et al. (2004) and Ernst et al. (2005), Bjork et al. (2004) found decreased accumbens activity relative to adults during a gain versus no gain contrast. Whereas Bjork et al. (2004) reported MR signal change over the entire experiment, we examined MR changes across the entire experiment and also during early and late trials, with the later trials showing greater activation in adolescents relative to adults.

A second difference in the current study, relative to the existing literature (O'Doherty et al., 2001, Elliott et al., 2003, Galvan et al., 2005), was the lack of a main effect of reward value in the OFC across subjects. In examining this main effect, we collapsed OFC activity across age groups and across the experiment. Other reward studies of the OFC have not included developmental populations, who have diffuse and more variable patterns of activity in this region (Casey et al., 1997). Inclusion of developmental populations thus increased the variability in recruitment of this region, with less consistent patterns of OFC activity. Furthermore, our data showed that, for later trials of the experiment, OFC activity differed for larger relative to smaller rewards, but showed a less precise mapping to reward value relative to the NAcc, which showed discrete patterns of activity to each reward value across age groups, consistent with our previous work (Galvan et al., 2005) and that of others (Elliott et al., 2003).

\section{Implications}

Our results suggest that there are protracted maturational changes in top-down control systems relative to subcortical regions implicated in appetitive behaviors. These different developmental trajectories may contribute to suboptimal choices in adolescents being driven more by appetitive systems than control systems (Spear, 2000). Understanding the development of structural and functional connectivity of reward-related mesolimbic circuitry may further inform the field on the neurobiological basis of increased reward-seeking and adolescent-onset addiction.

A neural framework similar to the one we propose here has been suggested to explain addiction. Accordingly, the PFC is "hijacked" by an impulsive subcortical system, which might render it unable to appropriately modulate decisions in the context of future consequences (Bechara, 2005). Our findings are consistent with this speculation but occur during typical development. Thus, disproportionate contributions of subcortical systems rel- ative to prefrontal regulatory systems may underlie poor decision-making that predisposes adolescents to drug use and, ultimately, addiction.

\section{References}

Bechara A (2005) Decision-making, impulse control and loss of willpower to resist drugs: a neurocognitive perspective. Nat Neurosci 8:1458-1463.

Bjork JM, Knutson B, Fong GW, Caggiano DM, Bennett SM, Hommer DW (2004) Incentive-elicited brain activation in adolescents: similarities and differences from young adults. J Neurosci 24:1793-1802.

Boynton GM, Engel SA, Glover GH, Heeger DJ (1996) Linear systems analysis of functional magnetic resonance imaging in human V1. J Neurosci 16:4207-4221.

Brown TT, Lugar HM, Coalson RS, Miezin FM, Petersen SE, Schlaggar BL (2005) Developmental changes in human cerebral functional organization for word generation. Cereb Cortex 15:275-290.

Bunge S, Dudukovic NM, Thomason ME, Vaidya CJ, Gabrieli JD (2002) Immature frontal lobe contributions to cognitive control in children: evidence from fMRI. Neuron 33:301-311.

Burgund ED, Kang HC, Kelly JE, Buckner RL, Snyder AZ, Petersen SE, Schlaggar BL (2002) The feasibility of a common stereotactic space for children and adults in fMRI studies of development. NeuroImage 17:184-200.

Casey BJ, Trainor R, Orendi J, Schubert A, Nystrom LE, Cohen JD, Noll D, Giedd J, Castellanos X, Haxby J, Forman SD, Dahl RE, Rapoport JL (1997) A pediatric functional MRI study of prefontal activation during performance of a Go-No-Go task. J Cogn Neurosci 9:835-847.

Casey BJ, Thomas KM, Davidson MC, Kunz K, Franzen P (2002) Dissociating striatal and hippocampal function developmentally with a stimulusresponse compatibility task. J Neurosci 22:8647-8652.

Casey BJ, Galvan A, Hare TA (2005) Changes in cerebral functional organization during cognitive development. Curr Opin Neurobiol 15:239-244.

Cetin T, Freudenberg F, Fuchtemeier M, Koch M (2004) Dopamine in the orbitofrontal cortex regulates operant responding under a progressive ratio of reinforcement in rats. Neurosci Lett 370:114-117.

Chudasama Y, Robbins TW (2003) Dissociable contributions of the orbitofrontal and infralimbic cortex to pavlovian autoshaping and discrimination reversal learning: further evidence for the functional heterogeneity of the rodent frontal cortex. J Neurosci 23:8771-8780.

Cox RW (1996) AFNI: software for analysis and visualization of functional magnetic resonance neuroimages. Comput Biomed Res 29:162-173.

Cox SM, Andrade A, Johnsrude IS (2005) Learning to like: a role for human orbitofrontal cortex in conditioned reward. J Neurosci 25:2733-2740.

Cromwell HC, Schultz W (2003) Effects of expectations for different reward magnitudes on neuronal activity in primate striatum. J Neurophysiol 89:2823-2838.

Davidson MC, Horvitz JC, Tottenham N, Fossella JA, Watts R, Ulug A, Casey B (2004) Differential cingulate and caudate activation following unexpected nonrewarding stimuli. NeuroImage 23:1039-1045.

Delgado MR, Miller M, Inati S, Phelps EA (2005) An fMRI study of rewardrelated probability learning. NeuroImage 24:862-873.

Dickinson A, Mackintosh N (1978) Classical conditioning in animals. Annu Rev Psychol 29:587-612.

Durston S, Davidson MC, Tottenham N, Galvan A, Spicer J, Fossella JA, Casey B (2006) A shift from diffuse to focal cortical activity with development. Dev Sci 9:1-8.

Duvernoy HM (1999) The human brain: surface, three-dimensional sectional anatomy with MRI and blood supply, Ed 2. New York: Springer.

Elliott R, Dolan RJ, Frith CD (2000) Dissociable functions in the medial and lateral orbitofrontal cortex: evidence from human neuroimaging studies. Cereb Cortex 10:308-317.

Elliott R, Newman JL, Longe OA, Deakin JFW (2003) Differential response patterns in the striatum and orbitofrontal cortex to financial reward in humans: a parametric functional magnetic resonance imaging study. J Neurosci 23:303-307.

Ernst M, Nelson EE, Jazbec SP, McClure EB, Monk CS, Leibenluft E, Blair J, Pine DS (2005) Amygdala and nucleus accumbens in responses to receipt and omission of gains in adults and adolescents. NeuroImage 25:1279-1291.

Forman SD, Cohen JD, Fitzgerald M, Eddy WF, Minton MA, Noll DC (1995) Improved assessment of significant activation in functional magnetic res- 
onance imaging (fMRI): use of a cluster-size threshold. Magn Reson Med 33:636-647.

Galvan A, Hare TA, Davidson M, Spicer J, Glover G, Casey BJ (2005) The role of ventral frontostriatal circuitry in reward-based learning in humans. J Neurosci 25:8650-8656.

Giedd JN, Blumenthal J, Jeffries NO, Castellanos FX, Liu H, Zijdenbos A, Paus T, Evans AC, Rapoport JL (1999) Brain development during childhood and adolescence: a longitudinal MRI study. Nat Neurosci 2:861-863.

Glover GH, Thomason ME (2004) Improved combination of spiral-in/out images for BOLD fMRI. Magn Reson Med 51:863-868.

Gogtay N, Giedd JN, Lusk L, Hayashi KM, Greenstein D, Vaituzis AC, Nugent III TF, Herman DH, Clasen LS, Toga AW, Rapoport JL, Thompson PM (2004) Dynamic mapping of human cortical development during childhood through early adulthood. Proc Natl Acad Sci USA 101:8174-8179.

Gottlieb D (2004) Acquisition with partial and continuous reinforcement in pigeon autoshaping. Learn Behav 32:321-334.

Gottlieb D (2005) Acquisition with partial and continuous reinforcement in rat magazine approach. J Exp Psychol Anim Behav Process 31:319-333.

Haber SN (2003) The primate basal ganglia: parallel and integrative networks. J Chem Neuroanat 26:317-330.

Haruno M, Kuroda T, Doya K, Toyama K, Kimura M, Samejima K, Imamizu H, Kawato M (2004) A neural correlate of reward-based behavioral learning in caudate nucleus: a functional magnetic resonance imaging study of a stochastic decision task. J Neurosci 24:1660-1665.

Hikosaka K, Watanabe M (2000) Delay activity of orbital and lateral prefrontal neurons of the monkey varying with different rewards. Cereb Cortex 10:263-271.

Hosokawa T, Kato K, Inoue M, Mikami A (2005) Correspondence of cue activity to reward activity in the macaque orbitofrontal cortex. Neurosci Lett 389:121-182.

Hyman SE, Malenka RC (2001) Addiction and the brain: the neurobiology of compulsion and its persistence. Nat Rev Neurosci 2:695-703.

Irwin J (1993) Adolescence and risk taking: how are they related. Newbury Park, CA: Sage.

Kang HC, Burgund ED, Lugar HM, Petersen SE, Schlaggar BL (2003) Comparison of functional activation foci in children and adults using a common stereotactic space. NeuroImage 19:16-28.

Knutson B, Adams CM, Fong GW, Hommer D (2001) Anticipation of increasing monetary reward selectively recruits nucleus accumbens. J Neurosci 21:1-5.

Kuhnen CM, Knutson B (2005) The neural basis of financial risk taking. Neuron 47:763-770.

Laviola G, Adriani W, Terranova ML, Gerra G (1999) Psychobiological risk factors for vulnerability to psychostimulants in human adolescents and animal models. Neurosci Biobehav Rev 23:993-1010.

Laviola G, Macri S, Morley-Fletcher S, Adriani W (2003) Risk-taking behavior in adolescent mice: psychobiological determinants and early epigenetic influence. Neurosci Biobehav Rev 27:19-31.

Luna B, Sweeney JA (2004) The emergence of collaborative brain function: fMRI studies of the development of response inhibition. Ann NY Acad Sci 1021:296-309.

Luna B, Thulborn KR, Munoz DP, Merriam EP, Garver KE, Minshew NJ, Keshavan MS, Genovese CR, Eddy WF, Sweeney JA (2001) Maturation of widely distributed brain function subserves cognitive development. NeuroImage 13:786-793.

May JC, Delgado MR, Dahl RE, Stenger A, Ryan ND, Fiez JA, Carter CS (2004) Event-related functional magnetic resonance imaging of rewardrelated brain circuitry in children and adolescents. Biol Psychiatry 55:359-366.

McClure SM, Laibson DI, Loewenstein G, Cohen JD (2004) Separate neural systems value immediate and delayed monetary rewards. Science 306:503-507.
Miller EK, Cohen JD (2001) An integrative theory of prefrontal cortex function. Annu Rev Neurosci 24:167-202.

Monk CS, McClure EB, Nelson EE, Zarahn E, Bilder RM, Leibenluft E, Charney DS, Ernst M, Pine DS (2003) Adolescent immaturity in attentionrelated brain engagement to emotional facial expressions. NeuroImage 20:420-428.

Moses P, Roe K, Buxton RB, Wong EC, Frank LR, Stiles J (2002) Functional MRI of global and local processing in children. NeuroImage 16:415-424.

O’Doherty J, Kringelbach M, Rolls ET, Hornak J, Andrews C (2001) Abstract reward and punishment representations in the human orbitofrontal cortex. Nat Neurosci 4:95-102.

O’Doherty J, Critchley H, Deichmann R, Dolan RJ (2003) Dissociating valence of outcome from behavioral control in human orbital and ventral prefrontal cortices. J Neurosci 23:7931-7939.

Pasupathy A, Miller EK (2005) Different time courses of learning-related activity in the prefrontal cortex and striatum. Nature 433:873-876.

Pecina S, Cagniard B, Berridge KC, Aldridge JW, Zhuang X (2003) Hyperdopaminergic mutant mice have higher "wanting" but not "liking" for sweet rewards. J Neurosci 23:9395-9402.

Rosenberg D, Lewis DA (1994) Changes in the dopaminergic innervation of monkey prefrontal cortex during late postnatal development: a tyrosine hydroxylase immunohistochemical study. Biol Psychiatry 36:272-277.

Rosenberg D, Lewis DA (1995) Postnatal maturation of the dopaminergic innervation of monkey prefrontal and motor cortices: a tyrosine hydroxylase immunohistochemical analysis. J Comp Neurol 358:383-400.

Rubia K, Overmeyer S, Taylor E, Brammer M, Williams S, Simmons A, Andrew C, Bullmore E (2000) Functional frontalisation with age: mapping neurodevelopmental trajectories with fMRI. Neursci Biobehav Rev 24:13-19.

Silveri MM, Tzilos GK, Pimentel PJ, Yurgelun-Todd DA (2004) Trajectories of adolescents emotional and cognitive development: effects of sex and risk for drug use. Ann NY Acad Sci 1021:363-370.

Sowell ER, Thompson PM, Holmes CJ, Jernigan TL, Toga AW (1999) In vivo evidence for post-adolescent brain maturation in frontal and striatal regions. Nat Neurosci 2:859-861.

Sowell ER, Peterson BS, Thompson PM, Welcome SE, Henkenius AL, Toga AW (2003) Mapping cortical change across the human life span. Nat Neurosci 6:309-315.

Spear LP (2000) The adolescent brain and age-related behavioral manifestations. Neurosci Biobehav Rev 24:417-463.

Steinberg L (2004) Risk taking in adolescence: what changes, and why? Ann NY Acad Sci 1021:51-58.

Talairach J, Tournoux P (1988) Co-planar stereotaxic atlas of the human brain. New York: Thieme.

Thomas KM, Hunt R, Vizueta N, Sommer T, Durston S, Yang Y, Worden MS (2004) Evidence of developmental differences in implicit sequence learning: an fMRI study of children and adults. J Cogn Neurosci 16:1339-1351.

Tremblay K, Kraus N, McGee T (1998) The time course of auditory perceptual learning: neurophysiological changes during speech-sound training. NeuroReport 9:3557-3560.

Tremblay L, Schultz W (1999) Relative reward preference in primate orbitofrontal cortex. Nature 398:704-708.

Tversky A, Kahneman D (1981) The framing of decisions and the psychology of choice. Science 211:453-458.

Volkow ND, Fowler J, Wang G, Swanson JM (2004) Dopamine in drug abuse and addiction: results from imaging studies and treatment implications. Mol Psychiatry 9:557-569.

Zald DH, Boileau I, El-Dearedy W, Gunn R, McGlone F, Dichter GS, Dagher A (2004) Dopamine transmission in the human striatum during monetary reward tasks. J Neurosci 24:4105-4112. 